\title{
Tunable low-temperature dissipation scenarios in palladium nanomechanical resonators
}

\author{
S. Rebari, ${ }^{1}$ Shelender Kumar, ${ }^{1}$ S. Indrajeet,,${ }^{1, *}$ Abhishek Kumar,,${ }^{1, \dagger}$ Satyendra P. Pal,,${ }^{1,+}$ D. Weiss, ${ }^{2}$ and A. Venkatesan ${ }^{1, \S}$ \\ ${ }^{1}$ Department of Physical Sciences IISER Mohali, Knowledge City, Sector 81, SAS Nagar, Manauli P.O. 140306, India \\ ${ }^{2}$ Experimental and Applied Physics, University of Regensburg, D-93040 Regensburg, Germany \\ (Received 5 January 2017; revised manuscript received 7 May 2017; published 20 June 2017)
}

\begin{abstract}
We study dissipation in palladium (Pd) nanomechanical resonators at low temperatures in the linear response regime. Metallic resonators have shown characteristic features of dissipation due to tunneling two-level systems (TLS). The system described here offers a unique tunability of the dissipation scenario by adsorbing hydrogen $\left(\mathrm{H}_{2}\right)$, which induces a compressive stress. The intrinsic stress is expected to alter TLS behavior. We find a sublinear $\sim T^{0.4}$ dependence of dissipation in a limited temperature regime. As seen in TLS dissipation scenarios, we find a logarithmic increase of frequency from the lowest temperatures till a characteristic temperature $T_{\mathrm{co}}$ is reached. In samples without $\mathrm{H}_{2}, T_{\text {co }} \sim 1 \mathrm{~K}$ was seen, whereas with $\mathrm{H}_{2}$ it is clearly reduced to $\sim 700 \mathrm{mK}$. Based on standard TLS phenomena, we attribute this to enhanced phonon-TLS coupling in samples with compressive strain. We also find that with $\mathrm{H}_{2}$ there is a saturation in low-temperature dissipation, which may possibly be due to super-radiant interaction between TLS and phonons. We discuss the data in the scope of TLS phenomena and similar data for other systems.
\end{abstract}

DOI: 10.1103/PhysRevB.95.214113

\section{INTRODUCTION:}

Nanoelectromechanical systems (NEMS) are not only sensitive transducers but also form an excellent platform to explore basic physical phenomena. The spectrum of phenomena include potential macroscopic quantum states [1], electronphonon coupling [2], and mechanospintronic phenomena [3]. Scaling of NEMS to smaller sizes (or higher frequencies) results in higher dissipation [4]. Typically, $\mathrm{GHz}$ frequency devices that can satisfy the rudimentary quantum condition $\hbar \omega \geqslant k_{B} T$ at dilution fridge temperatures are limited by intrinsic losses despite geometric aspects like clamping loss at boundaries are overcome by new resonator designs $[5,6]$. Photon pressure in microwave cavities can also squeeze $\mathrm{MHz}$ frequency NEMS, resulting in occupation numbers close to the ground state of a quantized harmonic oscillator [7]. A dilation-mode resonator is the only system so far to show evidence for macroscopic quantum behavior in mechanical systems [8]. Intrinsic loss mechanisms in NEMS are not yet fully understood and are crucial to understand decoherence of quantum phenomena [9].

\section{QUANTUM FRICTION DUE TO TUNNELING TWO-LEVEL SYSTEMS}

At temperatures typically below $4.2 \mathrm{~K}$ (boiling point of helium), the bulk elastic properties of most solids like Young's modulus and thermal contraction or expansion coefficients do not change significantly. Hence, one may naively expect most solid-state resonant structures (like cantilevers, beams, or vibrating wires, etc.) will show uninteresting behavior

\footnotetext{
*Present address: Department of Physics, Syracuse University, Syracuse, NY 13244, USA.

${ }^{\dagger}$ Present address: NEST, Istituto Nanoscienze-CNR and Scuola Normale Superiore, Piazza San Silvestro 12, 56127 Pisa, Italy.

${ }^{\ddagger}$ Present address: Department of Physics, Indian Institute of Technology,Hauz khas, New Delhi 110016 India.

${ }^{\S}$ Electronic address: ananthv@iisermohali.ac.in
}

in their mechanical response at these temperatures. On the contrary, the mechanical responses of solid-state mechanical resonators do vary vibrantly even at temperatures down to even below $T \leqslant 3 \mathrm{mK}$ [10]. Entities like defects play a critical role in dissipation at these temperatures. The classic discovery by Zeller and Pohl [11] showed that excess specific heat of amorphous solids can be explained by the presence of two-level excitations. A phenomenological model based on tunneling two-level systems (TLS) has been able to model a variety of low-temperature mechanical and thermal properties of bulk solids and are not limited to modeling amorphous solids alone [12-14]. The standard TLS models assume that phenomenologically two-level excitations are present and probability of going to higher levels are negligible at low temperatures. The tunneling process between the two levels is modified by coupling to quasiparticles, such as phonons or electrons, giving a unique behavior for different systems. Since tunneling is a quantum effect often dissipation due to TLS is also referred to as quantum friction. Some of the key parameters of a phenomenological TLS are shown schematically in Fig. 1. Apart from the parameters for an isolated TLS as shown in Fig. 1(a), the overall energy landscape of TLS, such as the distribution function of TLS energies $\bar{P}\left(E, \Delta_{0}\right)$, distribution of typical relaxation times $\tau$ for tunneling (both $\bar{P}\left(E, \Delta_{0}\right.$, and $\tau$ are functionally related), and how the TLS interact with each other and with quasiparticles like phonons, gives rise to unique behavior for various classes of systems. These models have been successful in explaining many experimentally accessible properties of bulk solids both amorphous and crystalline. The experimentally studied properties usually have a Kramers-Kroning-type dispersion for a generalized susceptibility $\chi=\chi_{r}+i \chi_{\text {im }}$ containing a real (dissipative) and imaginary (dispersive) response, the dissipation $Q^{-1}$, and relative frequency shift $d f / f_{0}$ for measurements in this work. TLS models for amorphous glasses are general enough to be mapped to several crystalline and polycrystalline systems. TLS candidates for polycrystals are grain boundary angles, dislocations kinks and jogs, or other imperfections but with different energy scales for parameters like phonon-TLS 

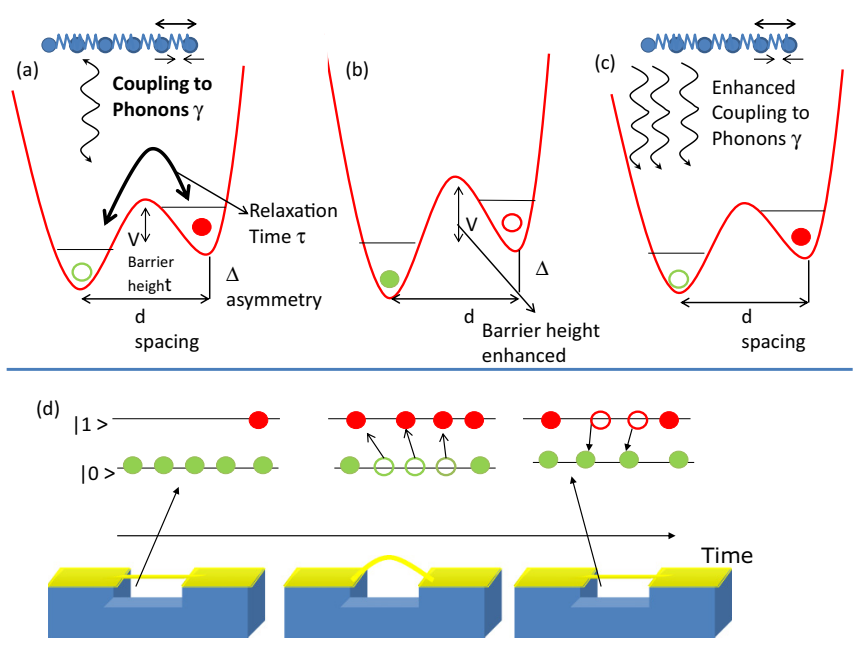

FIG. 1. (a) Schematic of a TLS. The key parameters are barrier height $V$, barrier asymmetry $\Delta$, relaxation time $\tau$, and the TLS-phonon coupling parameter $\gamma$. The tunnel amplitude $\Delta_{0} \sim$ $\hbar \Omega e^{-\lambda}$, where $\lambda=d \sqrt{2 m V / \hbar^{2}}$ and $m$ is a particle mass like term. TLS splitting energy $E^{2}=\Delta^{2}+\Delta_{0}^{2}$. Possible changes in TLS parameters (b) increase of barrier height (c) enhanced phonon coupling. (d) Schematic showing a random configuration of occupied two level system $|0\rangle$ and $|1\rangle$. As time proceeds the flexural motion couples to the TLS with relaxation time $\tau$ comparable to device frequency $1 / \omega$ and causes them to absorb or dissipate energy.

coupling, density of TLS, etc. Perfectly crystalline materials can show spin-glass states in their magnetic ordering. As an analogy, one may view TLS scenarios describing crystalline materials as a pseudospin glass [15]. TLS models predict a range of values for quantities like mechanical dissipation, e.g., $Q^{-1} \sim 10^{-3}-10^{-5}$ for amorphous dielectrics with some exceptions in stressed systems like silicon nitride [16]. Although TLS models have been successful in explaining the behavior of various bulk solids, there are still open problems, like probing TLS at extremely low and high frequencies. What are the physical attributes of TLS? How do we tune them? These are some of the open questions [17,18]. Some recent works in this direction are Ref. [19] on hydrogenated silicon altering the amorphous network and density-dependent voids in silicon [20].

In mesoscopic systems, the surface-to-volume ratio and size of the system complicate modeling them with established theories for bulk. Hybrid NEMS, i.e., semiconductor beams with metal electrodes to actuate in systems such as gallium arsenide, silicon, and diamond have shown extensive evidence for TLS phenomena [21]. One often ignores the effect of the electrodes like gold as negligible due to its smaller Young's modulus compared to the material under study. Recently, Al electrodes on silicon structures showed a profound difference when measured in the superconducting and nonsuperconducting state [22].

Stand alone metallic nanomechanical systems are simpler systems to study dissipation. It was demonstrated that at cryogenic temperatures tensile stress in these systems increases the quality factor (Q-factor) [23]. Metallic nanomechanical resonators made of gold [24] and aluminium [25,26] have shown clear evidence for TLS mechanisms. The behavior of metallic systems have been similar to amorphous dielectrics with electrons not playing a prominent role. For example, in Refs. [25] and [26], aluminum beams in normal and superconducting states show similar behavior as opposed to silicon devices with aluminium electrodes [22]. Metallic thin films and deformed bulk aluminium have been extensively studied in the context of TLS dissipation in Ref. [27]. In Ref. [28], Ag and Al films were studied as examples of normal and superconducting films. The electronic mean free path dependence of TLS dissipation in various polycrystalline metals were studied in Ref. [29]. Some of the key results obtained in these systems are also discussed along with the results in this work.

\section{SYSTEM UNDER STUDY}

In this work, we report our studies on mesoscopic $\mathrm{Pd}$ beams. The motivation for studying $\mathrm{Pd}$ is to probe a system where dissipation scenarios may be modified significantly intrinsically without external dissipation dilution [30] mechanisms like poor coupling to the environment or measurement system. Palladium's affinity to adsorb $\mathrm{H}_{2}$ is well known. In Ref. [31], nanoscale Au-Pd beams have been used as hydrogen sensors by probing frequency shifts due to adsorbed $\mathrm{H}_{2}$. The $\mathrm{H}_{2}$ not only covers the surface but also forms $\mathrm{H}^{+}$ions that diffuse inside and occupy interstitial sites in the $\mathrm{Pd}$, resulting in a compressive strain of the Pd lattice structure. Compressive or tensile stress can affect the barrier height $V$ as sketched in Fig. 1(b) or TLS-phonon coupling constant $\gamma$ as represented in Fig. 1(c). Metallic beams at cryogenic temperatures have intrinsic tensile stress due to differential thermal contraction with respect to the substrates. Our goal is to tune this tensile stress by exposure to $\mathrm{H}_{2}$, thereby modifying the TLS scenario. Intrinsic tensile stress is known to drastically alter TLS in systems like silicon nitride [16,30]. If TLS are seen as pseudospins, the internal stress plays the role of a pseudo-Zeeman term. One can expect any of the scenarios as sketched in Figs. 1(b) and 1(c) and possibly strong interactions among TLS. To the best of our knowledge, this is the only work on submicron metallic doubly clamped beams where the TLS properties are modified in one and the same material. We observe regimes of stronger TLS-phonon coupling as well as possible super-radiant TLS-phonon losses at very low temperatures.

Typical samples had a length $(l)$ of $4-5 \mu \mathrm{m}$, thickness $(t)$ around $80 \mathrm{~nm}$, and a width $(w)$ of $400-470 \mathrm{~nm}$. The beams were fabricated by performing e-beam lithography on $\mathrm{Si} / \mathrm{SiO}_{2}$ wafers to define a standard PMMA based lift-off stencil. The $\mathrm{Pd}$ films were deposited in an electron gun evaporator at $\sim 1.6 \AA /$ s to $2.0 \AA /$ s followed by a lift-off process in solvents to define the beams. The $\mathrm{SiO}_{2}$ was etched in buffered oxide etch (i.e., stabilized hydrofluoric acid etch solution). The etchant attacks the underlying $\mathrm{SiO}_{2}$ isotropically. The larger Pd contact areas are slightly undercut while the narrower regions are completely undercut in the etch process causing the beam to be suspended. Figure 2(a) illustrates the zoomed out image of a sample. A zoomed image of the undercut beam (samples B1 and B2) with pads is shown in Fig. 2(b). The samples were bonded by mechanically pressing indium coated gold 


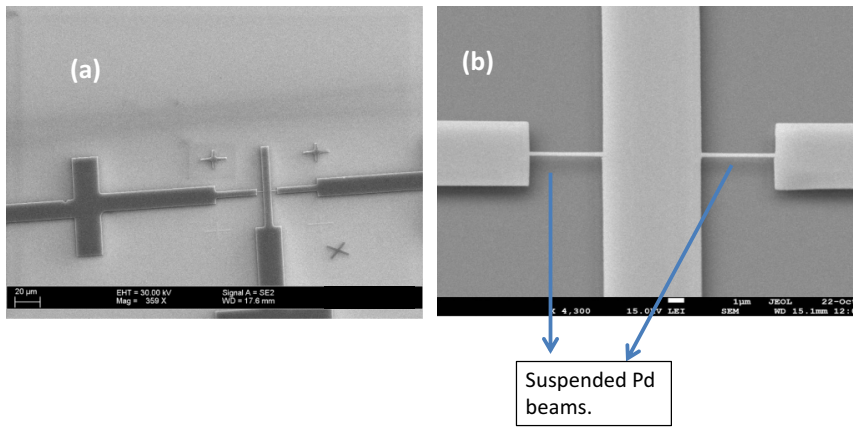

FIG. 2. (a) Overview of the etched beam structure. One of the markers was released and displaced during the wet etch process. (b) Magnification of the central area of samples B1 and B2 showing the two suspended beams.

wires on to the chip and to microstrip tracks on printed circuit boards.

\section{EXPERIMENTAL SETUP}

A schematic of the experimental setup is shown in Fig. 3(a). The experiments were carried out in a cryofree dilution fridge with a common vacuum space to isolate various cold stages. A separate brass vacuum can with homemade radio frequency (RF) feed-through was used to to let the $\mathrm{H}_{2}$ exchange gas into the sample space and avoid thermal linking of other stages of the fridge. The input lines were $50 \Omega$ cupronickel coaxial cables. Cryogenic 10-dB attenuators were placed at various cold stages giving a total of $\sim 40 \mathrm{~dB}$ in each of the input lines along. The output line had a $1-\mathrm{dB}$ attenuator to minimize reflections followed by room temperature preamplifiers. Biastees at the last stage were used to protect the samples from static.

We used a standard magnetomotive technique to probe the resonant response. RF current from a vector network analyzer was driven through the sample with a magnetic field parallel to the wafer plane to excite and detect out-of-plane motion of the beam due to the Lorentz force [32].

The transmission parameters $S_{21}$ were used to obtain the real and imaginary part of the response. The response at finite magnetic field has additional damping due to eddy-currentinduced damping and external impedances resulting in a loaded quality factor. A Lorentzian fit to the real and imaginary part of the resonant response was used to extract the loaded Qfactor $Q_{L}$ and resonant frequency $f_{L}$. As expected in standard magnetomotive technique, the eddy current damping showed a linear dependence for $B^{2}$ versus $Q_{L}^{-1}$ in all cases satisfying the relation $Q_{L}^{-1}=Q_{0}^{-1}\left(1+\alpha B^{2}\right)=Q_{0}^{-1}\left[1+\frac{R_{m} \operatorname{Re}\left(Z_{\text {ext }}\right)}{\left|Z_{\text {ext }}^{2}\right|}\right]$, where $R_{m}=\frac{\epsilon l^{2} B^{2} Q_{0}}{2 \pi f_{0} m}$ is the mechanical equivalent of resistance depending on resonator parameters (length, frequency, mass, and intrinsic Q-factor $Q_{0}$ ). The frequency squared also showed a quadratic dependence in field with $f_{l}^{2}=f_{o}^{2}+\beta B^{2}$ due to the presence of reactive components, like a bias-tee that protected the samples from static discharges. The term $\beta$ is given by $\frac{Z_{c} \operatorname{Im}\left(Z_{\text {ext }}\right)}{\left|Z_{\text {ext }}^{2}\right|}$, where $Z_{c}$ is the characteristic impedance of the measurement system $50 \Omega$ in this case. A typical field-dependent amplitude response for one of the devices sample A1 is shown in Fig. 3(b). One clearly sees an increase in signal at higher magnetic fields due to induced electromotive force and at the same time a broadening of the peaks due to the eddy-current-induced damping. The linear fits to the loaded quality factor $Q_{L}$ and frequency squared $f_{L}^{2}$ is shown in Figs. 3(c) and 3(d).

Since Pd samples broke easily on thermal cycling in initial trials, two sets of similar samples were investigated with and without addition of hydrogen. Sample set A consists of two beams forming an RF bridge. Sample A1 of dimensions $(4.5 \mu \mathrm{m} \times 430 \mathrm{~nm} l \times w)$ and A2 $(4.5 \mu \mathrm{m} \times 470 \mathrm{~nm} l \times w)$ were studied without hydrogen. The samples were pumped down to below $\sim 4 \times 10^{-5}$ Torr for over one day with a turbo pump equipped with a cold trap to remove any unintentional hydrogen. It was continuously pumped during cooling down till a temperature less than $10 \mathrm{~K}$ was reached. Sample A1 had a resonant frequency of $19.04 \mathrm{MHz}$, magnetomotive damping parameters $\alpha \sim 1.33 \times 10^{-6} / T^{2}$ and $\beta \sim-2.22 \times$ $10^{9} \mathrm{~Hz}^{2} / T^{2}$. The second sample A2 had a resonant frequency of $\sim 29.2 \mathrm{MHz}$ with the magneto-motive damping parameters $\alpha \sim 1.19 \times 10^{-6} / T^{2}$ and $\beta-4.55 \times 10^{9} \mathrm{~Hz}^{2} / T^{2}$. Although both sample dimensions were comparable, sample A2 trapped some indium in the under-etched region, thereby reducing its effective length to $\sim 3.5 \mu \mathrm{m}$, thus increasing the frequency. An electron microscope image taken after measurement along with an energy dispersive spectroscopy scan confirmed the presence of indium. The first sample showed some buckling that possibly explains a reduced frequency from estimates by $\sim 15 \%$.

Sample set B consisted of two samples B1 $(\sim 4.35 \mu \mathrm{m} \times$ $390 \mathrm{~nm} l \times w)$ and B2 $(\sim 4.35 \mu \mathrm{m} \times 366 \mathrm{~nm} l \times w)$ forming a RF bridge. This sample set was subject to two cool downs with different initial pressures of exchange gas of $\mathrm{H}_{2}$.

In the first cool down after reaching high vacuum, $\mathrm{H}_{2}$ exchange gas of $\sim 2 \times 10^{-3}$ Torr was introduced and subsequently pumped to lower $10^{-4}$ Torr when the mixing chamber temperature was below $160 \mathrm{~K}$. In the first exposure to $\mathrm{H}_{2}$, the sample $\mathrm{B} 1$ had a resonant frequency of $f \sim 20.27 \mathrm{MHz}$ and the magnetomotive damping parameters $\alpha \sim 1.05 \times 10^{-6} / T^{2}$ and $\beta \sim-2.42 \times 10^{9} \mathrm{~Hz}^{2} / T^{2}$. Sample B2 had a resonant frequency $f_{0} \sim 19.3 \mathrm{MHz}$ and magnetomotive damping parameters $\alpha \sim 1.43 \times 10^{-6} / T^{2}$ and $\beta \sim-2.31 \times 10^{9} \mathrm{~Hz}^{2} / T^{2}$.

Sample set B was subject to a second cool-down. After warming to room temperature and reaching high vacuum once again, an initial $\mathrm{H}_{2}$ exchange gas at a pressure $\sim 10^{-2}$ Torr was introduced and subsequently pumped to lower $10^{-4}$ Torr when the mixing chamber temperature was below $160 \mathrm{~K}$. Sample B1 retained similar resonant frequency $f_{0} \sim 20.35 \mathrm{MHz}$ and the magnetomotive parameters were $\alpha \sim 1.16 \times 10^{-6} / T^{2}$ and $\beta \sim-2.52 \times 10^{-9} \mathrm{~Hz}^{2} / T^{2}$. During this cool down, B2 was heated with a $0.5 \mu \mathrm{A}$ low-frequency current from room temperature down to $160 \mathrm{~K}$. This was unstable in frequency possibly due to excessive adsorption of $\mathrm{H}_{2}$ causing additional diffusion-induced dissipation [33,34], and the data is not discussed here.

The field dependence at a fixed temperature can be used to estimate the intrinsic $Q_{0}$ and frequency $f_{0}$ from loaded values measured at $4 T$ [24]. It is not possible to know exactly how stress relaxes in these beams and the mode shape the beam 
(a)
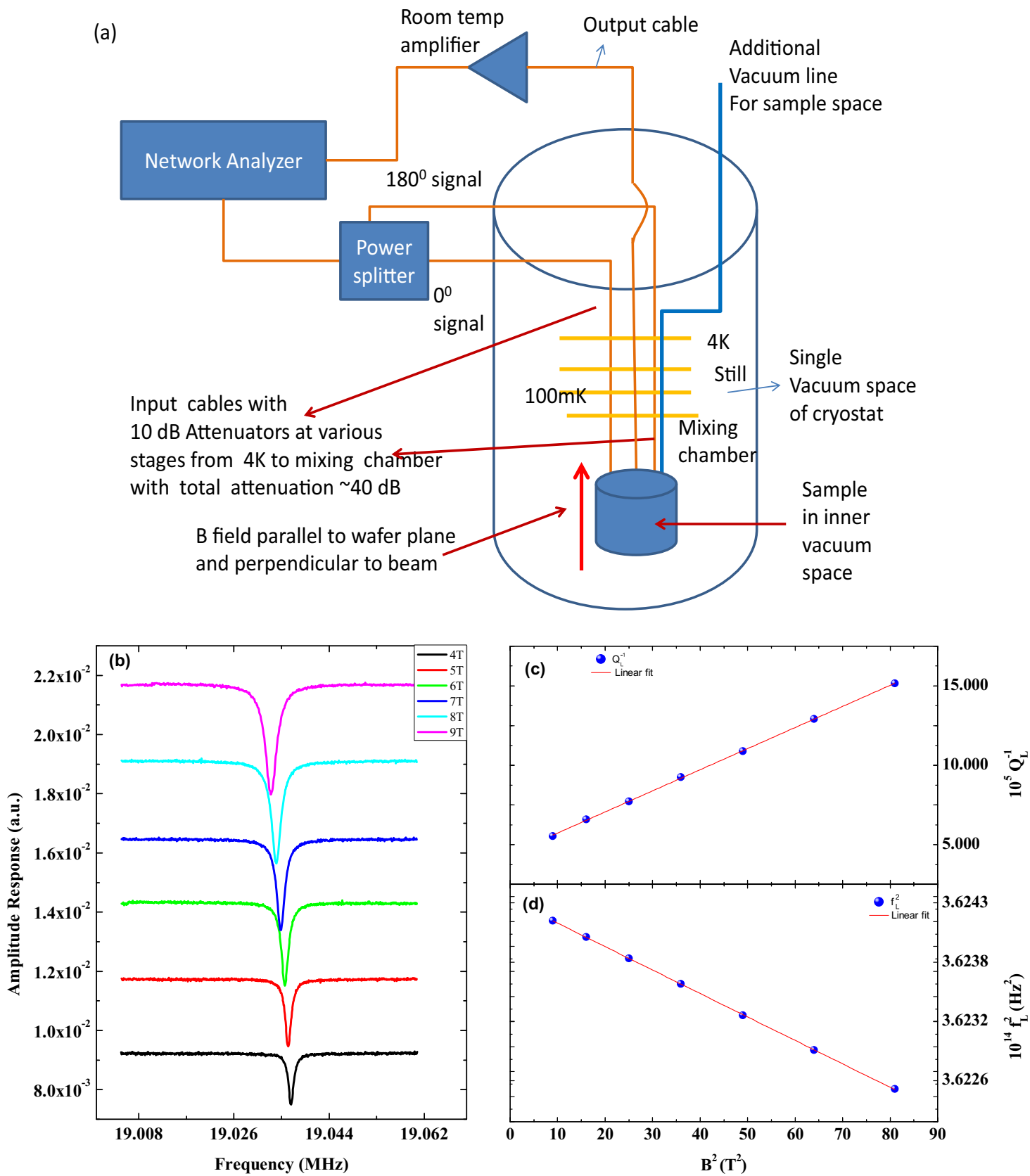

FIG. 3. (a) Schematic of the measurement setup. (b)Amplitude response of a sample as a function of B field sample A1. (c) A linear fit to $B^{2}$ vs $Q_{L}^{-1}(\mathrm{~d})$ a linear fit to $B^{2}$ vs $f_{0}^{2}$. The data is taken at $T \sim 160 \mathrm{mK}$.

takes at cryogenic temperatures. All beams showed very small buckling after the cool down. There was no significant change in the order of magnitude of the magnetomotive damping parameter $\alpha$ when $\mathrm{H}_{2}$ was added with all samples and was nonmonotonic with $\mathrm{H}_{2}$. The most important change was the drive power required to tune the system into the linear response regime was $\sim-90 \mathrm{dBm}$ (when precooled with $\sim 2 \times 10^{-3}$ Torr of $\mathrm{H}_{2}$, hereafter referred to as low $\mathrm{H}_{2}$ ) and $\sim-110 \mathrm{dBm}$ (when precooled with $\sim 10^{-2}$ Torr $\mathrm{H}_{2}$, hereafter referred to as high $\mathrm{H}_{2}$ ) and $\sim-80 \mathrm{dBm}$ when cooled without $\mathrm{H}_{2}$. These power levels mark the onset of a non-Lorentzian shape or change of frequency when driven $\sim 5 \mathrm{~dB}$ above this power. A power of $\sim 5 \mathrm{~dB}$ is chosen as a conservative estimate. The reduced power levels needed to tune the $\mathrm{H}_{2}$-loaded samples to the linear regime clearly indicates softening of the beams with $\mathrm{H}_{2}$ due to additional compressive stress.

\section{RESULTS AND DISCUSSION}

A few sample amplitude responses of sample B1 are given in Fig. 4, where panels 4(a) and 4(b) are below and above a characteristic temperature, $\sim 700 \mathrm{mK}$ in this case. The direction of arrows indicate increase of temperature in Figs. 4(a) and 4(b). In both cases, as the temperature is raised, the amplitude drops and the line-width broadens, as one would expect to see when a resonant structure like a string is heated. In Fig. 4(a), one observes a counterintuitive increase of resonant frequency $f_{0}$ with temperature. One typically expects 


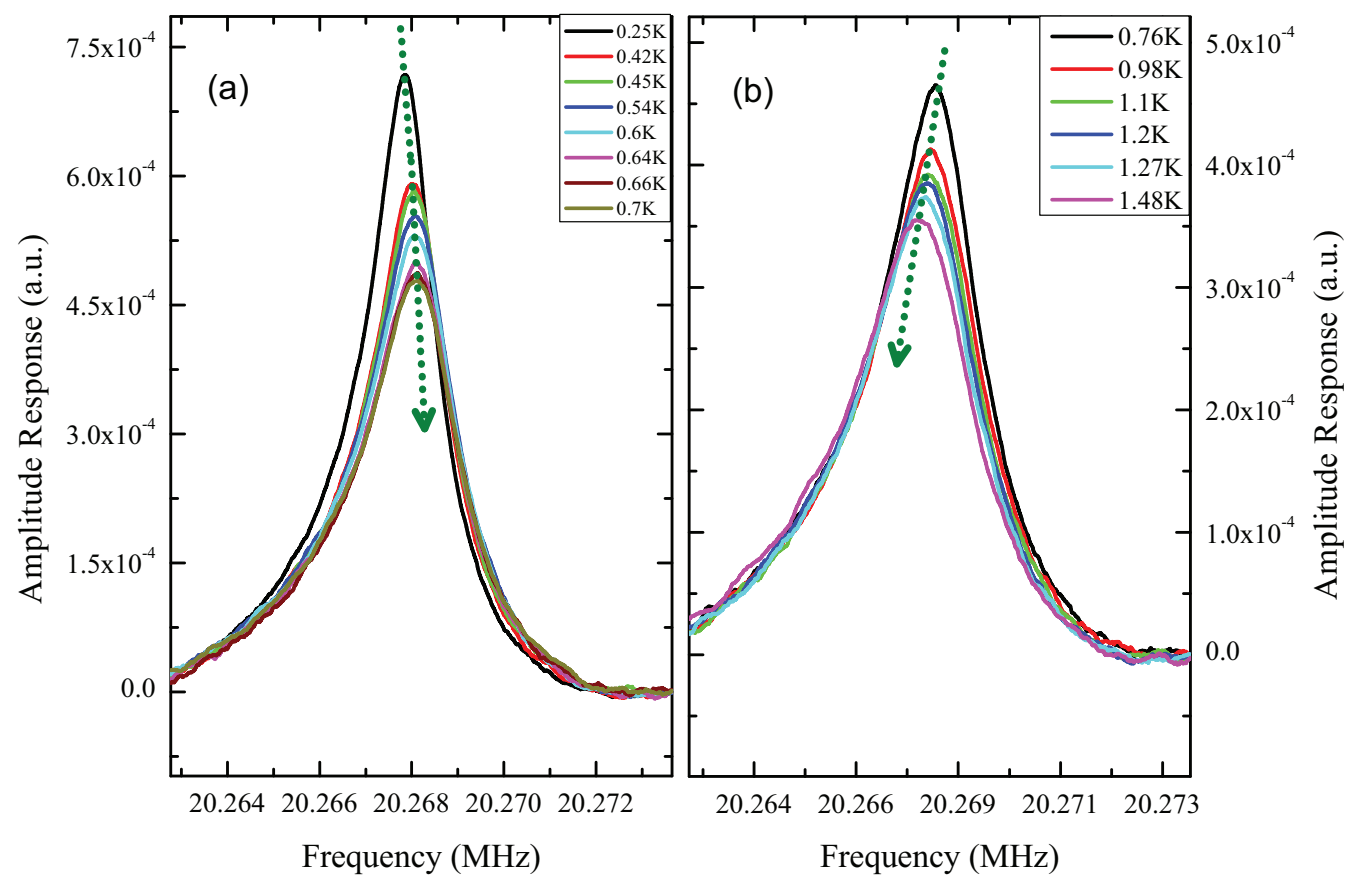

FIG. 4. Smooth fits to temperature-dependent amplitude response at $B=4 T$ for sample B1 at two temperature regimes (a) below $\sim 700 \mathrm{mK}$ (b) above $\sim 700 \mathrm{mK}$. The arrow points toward increasing temperature. In (a) the frequency increases with temperature, whereas in (b) it decreases with temperature.

an increase in temperature to soften materials and reduce the frequency. As mentioned in the introduction changes of mechanical properties or thermal contraction effects are negligible at these temperature scales. This increase in frequency with temperature is a classic signature of TLS dissipation phenomena. Once one crosses the characteristic temperature $T_{\text {co }} \sim 700 \mathrm{mK}$, the resonance frequency in Fig. 4(b) decreases as usual with increasing temperature.

In line with the generalized susceptibility of TLS phenomenology $\chi=Q^{-1}+i \frac{d f}{f_{0}}$, the data sets of dissipation and relative frequency shift (with reference to a reference frequency $f_{0}$ at an arbitrary temperature $T_{0}$ ) are presented side by side in Figs. 5-7.

The data for dissipation and relative frequency shift in the absence of $\mathrm{H}_{2}$ for $\mathrm{A} 119 \mathrm{MHz}$ and A2 $29 \mathrm{MHz}$ samples are presented in Fig. 5 .The relative frequency shift Fig. 5(a) shows a logarithmic increase of the form $\left(\frac{d f}{f_{o}}\right)=C \ln \left(\frac{T}{T_{o}}\right)$ till a characteristic temperature $T_{\text {co }} \sim 1 \mathrm{~K}$ is reached in both samples and the slope becomes negative beyond this point. The slope $C$ before $T_{\text {co }}$ is $C=1.85 \times 10^{-5}$ for A1 $19 \mathrm{MHz}$ sample and $3.5 \times 10^{-5}$ for A2 the $29 \mathrm{MHz}$ sample.

One may ascribe a characteristic relaxation time $\omega_{0} \tau \sim 1$ to this temperature, where $\omega_{0}$ is the device resonant frequency. In the absence of electrons in dielectric glasses, this may be interpreted as crossover from a resonant TLS mechanisms at low temperature $\omega_{0} \tau \geqslant 1$ to a relaxation dominant regime $\omega_{0} \tau \leqslant 1$. In dielectric glasses, the ratio of the slope of frequency shift above and below $T_{\text {co }}$ is expected to be $\sim 1.5$. The ratio of slopes of frequency shift above and below the crossover temperature is $\sim 2$ for sample A1 and 2.5 for sample A2 as opposed to 1.5 for dielectric glasses. In some systems like metallic glasses, $T_{\mathrm{co}}$ is an effect of phononic as well as of electronic mechanisms. This also produces unique features, like a linear and not logarithmic dependence of frequency shift above $T_{\text {co }}$ [35], which is not seen in our system.

In Ref. [29], several bulk polycrystalline metals, including $\mathrm{Pd}$, were studied and showed no dependence of the TLS coupling constant $\gamma$ on electronic mean free path. Since $\gamma$ was around $\sim 1 \mathrm{eV}$ independent of mean free path the same class of TLS as dielectric glasses was concluded to describe polycrystalline metals. In polycrystalline $\mathrm{Pd}$, a $T_{\text {co }} \sim 60 \mathrm{mK}$ was reported [29]. In the same work [29], an increase of $T_{\text {co }}$ by $50 \mathrm{mK}$ for annealed Pt and Ta was interpreted as a decrease in the TLS-phonon coupling constant $\gamma$ by using the standard frequency scaling relation for TLS $\omega \sim \gamma^{2} T_{\mathrm{co}}^{3}$. Annealing is usually expected to increase the tensile stress. Since our beams are under extreme tensile stress compared to bulk metals films, we may conclude that $\gamma$ is reduced. Overall, the behavior shows features of glass-like TLS models but does not fully fit either a dielectric glass or a metallic glass.

The dissipation $Q^{-1}$ in Fig. 5(b) shows two distinct regions a sublinear dependence $T^{m}$ with $m \sim 0.4$ below the $T_{\text {co }}$ and a saturation well above $T_{\text {co }}$. One can extrapolate the power law above $T_{\text {co }}$ as shown in Fig. 5(b) one cannot ignore the scatter in the $Q^{-1}$ data above $T_{\mathrm{co}}$. We only quote $T^{0.4}$ as an empirical average. The saturation in dissipation well above $T_{\text {co }}$ is associated with the regime where $\omega \tau<1$, where a spectrum of TLS with different relaxation times are excited.

High-temperature saturation in TLS phenomenology is related to the TLS density of states and interaction strength with phonons by a constant $C=P \gamma^{2} / E$, where $P$ is TLS density of states, $\gamma$ TLS-phonon coupling, and $E$ the Young's modulus. The constant is two to three times larger than the value estimated from the frequency shift data. A similar behavior has been seen for gold [24]. 


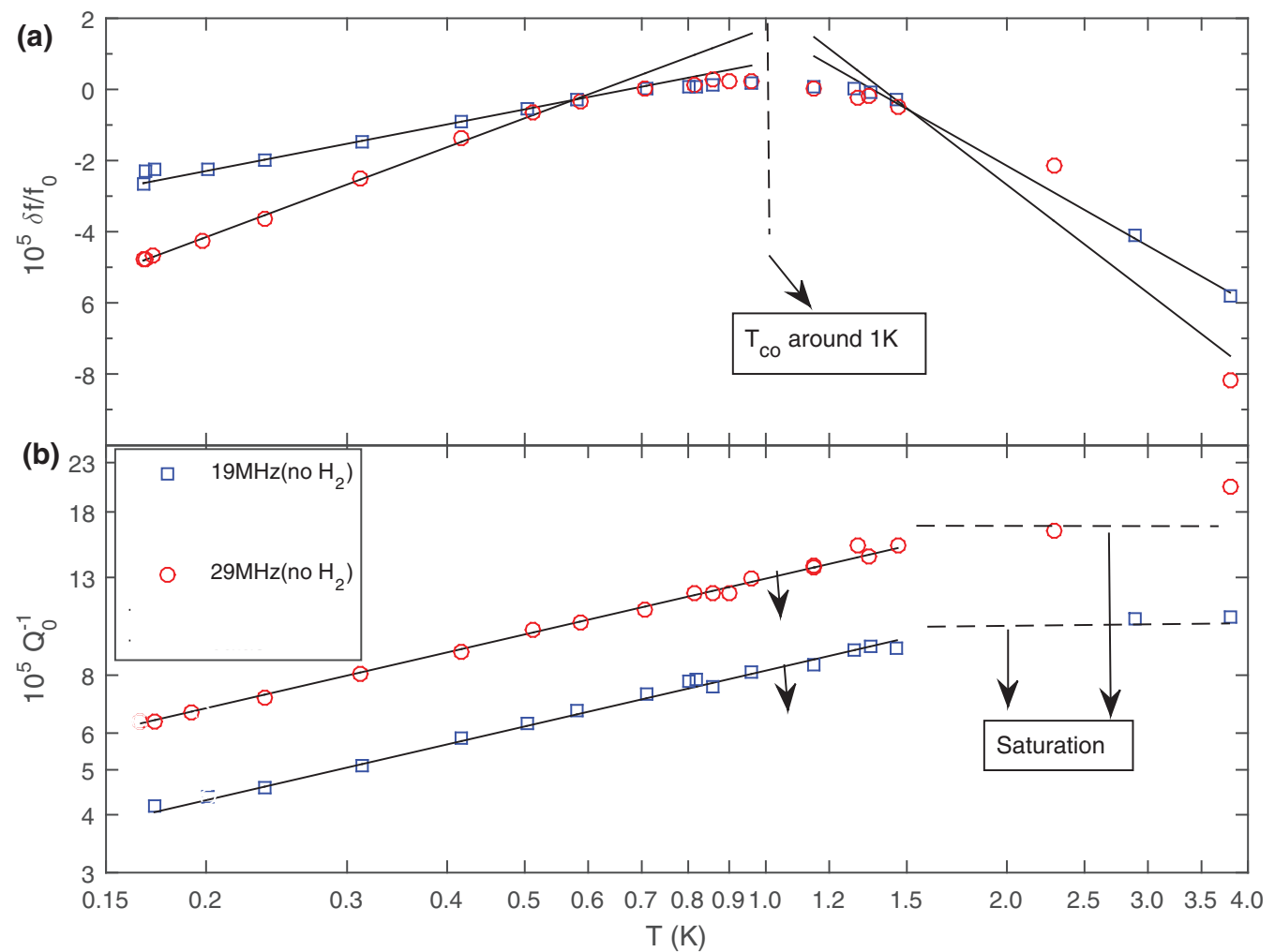

FIG. 5. Data in the $\mathrm{H}_{2}$ free samples $\mathrm{A} 1$ and A2. (a) Relative frequency shift $\frac{d f}{f_{0}}$ in these samples as a function of temperature. The logarithmic slope below $T_{\text {co }} \sim 1 \mathrm{~K}$ yields the parameter $C \sim 1.85 \times 10^{-5}$ for A1 (19 MHz sample) and $C \sim 3.5 \times 10^{-5}$ for A2 (29 MHz sample). (b) Damping $Q^{-1}$ as a function of temperature. Sublinear fits $\sim T^{0.39}$ for A1 (19 MHz sample) and $\sim T^{0.41}$ for A2 (29 MHz sample) are shown as a guide. The sublinear fit describes the behavior above $T_{\text {co }}$ with visibly very small deviations indicated by an unlabeled arrow. A saturation is seen at very high temperatures well above $T_{\mathrm{co}}$.

The data of the samples with low $\mathrm{H}_{2}$ concentration and the one with higher concentration are shown in Figs. 6 and 7, respectively. The behavior is qualitatively similar but shows essential differences from the hydrogen-free samples. For both low and high $\mathrm{H}_{2}$ concentrations, the samples showed a clear lowering of $T_{\text {co }}$ to around $700 \mathrm{mK}$. It is important to note that in our two devices with similar resonance frequency of $\sim 20$ $\mathrm{MHz}$, we see a lower crossover temperature compared to a device not exposed to $\mathrm{H}_{2}$ (compared to the $\mathrm{H}_{2}$ free case where the device showed $\left.T_{\mathrm{co}} \sim 1 \mathrm{~K}\right)$.

The dissipation $Q^{-1}$ shows a sublinear power law with $m$ in the range $0.37-0.43$ below $T_{\text {co }}$ and with no systematic dependence on $\mathrm{H}_{2}$ concentration. Hence, we quote an empirical mean of $\sim T^{0.4}$. There were no Debye peaks [34] in the dissipation shown in Figs. 6(a) and 7(a), indicating that $\mathrm{H}_{2}$ diffusion-induced mechanisms are not prominent at these concentrations and temperatures, which are also well below the freezing point of $\mathrm{H}_{2}$. One remarkable feature with the exposure to $\mathrm{H}_{2}$ is a low-temperature saturation in the dissipation $Q^{-1}$ below $200 \mathrm{mK}$ in Fig. 6 for both samples and in Fig. 7 below $300 \mathrm{mK}$. This feature is discussed separately in the last section.

In comparison with other systems, $\sim T^{0.5}$ was seen for gold [24] and a linear one $\sim T[25,26]$ for aluminium. The linear dependence of dissipation in $\mathrm{Al}$ in Ref. [25] was ascribed to one-dimensional phonon modes interacting with TLS at temperatures well below $1 \mathrm{~K}$.
A $T^{0.5}$ dependence has been predicted for dielectrics [36]. Although the sublinear power we observe may be close to the prediction in Ref. [36], we leave our arguments on an empirical basis as the theory applies to unstressed dielectric beams in a simple Euler bending beam limit. We have not attempted to apply scaling arguments [18] as the behavior in $H_{2}$ exposed, and $\mathrm{H}_{2}$ free beams are slightly different in terms of parameters like $T_{\text {co. }}$.

The overall magnitude of dissipation is not significantly lower in the $\mathrm{H}_{2}$-exposed samples, despite the observed softening. In bulk systems studied before, like strained aluminium, the magnitude of dissipation was seen to increase with strain, indicating more defects are introduced into the system [27]. The dimensionless constant $C$ in TLS phenomenlogy,

$C=\left\{\begin{array}{ll}\text { if } T<T_{\text {co }} & \text { from slope of }\left(\frac{d f}{f_{0}}\right) \sim 1.5 \text { to } 3.5 \times 10^{-5} \\ \text { if } T>T_{\text {co }} & \left(\frac{2}{\pi}\right) Q^{-1} \sim \frac{P \gamma^{2}}{E} \sim 3 \text { to } 9 \times 10^{-5}\end{array}\right.$,

is of similar order in the $\mathrm{H}_{2}$-free and the low- $\mathrm{H}_{2}$ scenario. In samples with resonant frequency close to $20 \mathrm{MHz}$, the constant $C$ from the slope of frequency shift has a small spread in the range 1.54-1.9 $\times 10^{-5}$ with no systematic dependence on $\mathrm{H}_{2}$ exposure. Only the $29 \mathrm{MHz}$ sample not exposed to $\mathrm{H}_{2}$ had $C \sim 3.5 \times 10^{-5}$. Consistently, $C$ estimated from $Q^{-1}$ at the saturation at high temperatures (or highest temperature data available) is two to three times higher than estimates from the frequency shift a behavior seen in gold beams also. 


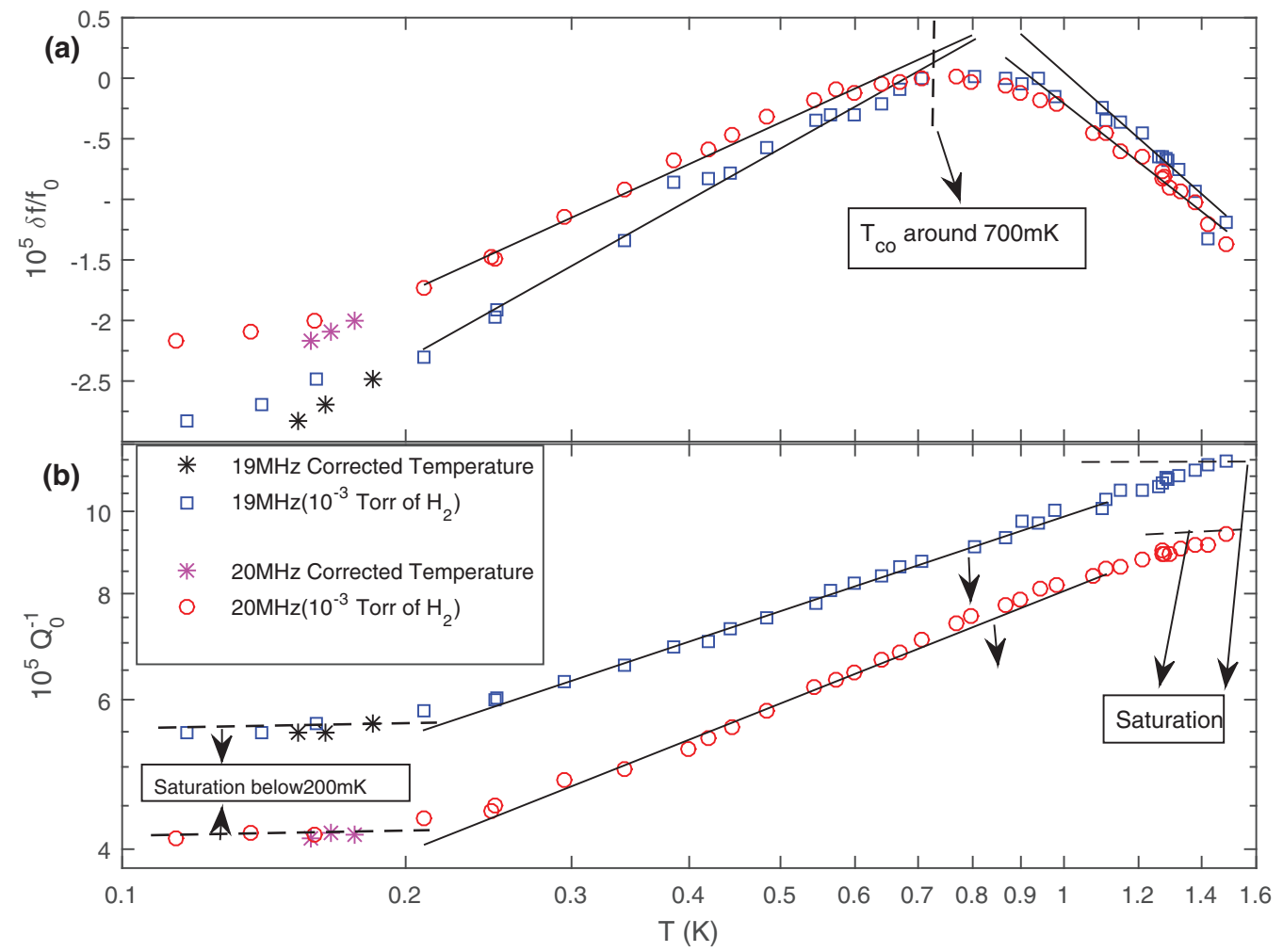

FIG. 6. Data from samples $\mathrm{B} 1$ and $\mathrm{B} 2$ when exposed to low concentration of $\mathrm{H}_{2}$. (a) $\left(\frac{d f}{f_{0}}\right)$ as a function of temperature are shown for these samples. The logarithmic slope below $T_{\text {co }} \sim 700 \mathrm{mK}$ yields the parameter $C$ to be $1.9 \times 10^{-5}$ for B1 $(20 \mathrm{MHz}$ sample $)$ and $1.6 \times 10^{-5}$ for B2 (19 MHz sample). (b) The dissipation $Q^{-1}$ as a function of temperature for the same two samples. A sublinear fit with $\sim T^{0.43}$ for B1 (20 MHz sample) and $\sim T^{0.37}$ for B2 $19 \mathrm{MHz}$ sample are shown as a guide. The unlabeled arrows indicate a small deviation above $T_{\text {co }}$ in the sublinear

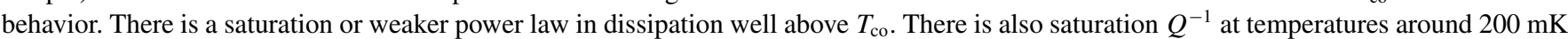
and below. The lowest three points show a small deviation from the logarithmic behavior shown in (a). The log fit at higher temperature data is used to correct the temperature of these points. The corrected temperature data are shown by $*$ symbols as opposed to the raw data in open circles or squares. The temperature corrected $Q^{-1}$ in (b) shown as a $*$ also retains the low-temperature saturation.

The similarity in value of $C$ is surprising considering the effective Young's modulus $E$ has to be lower in the case of the $\mathrm{H}_{2}$ scenario as reckoned by lower drive power to keep the response linear. The overall value of dissipation is also not high, indicating no new defects or TLS density being altered drastically. The only plausible explanation of getting a similar order of magnitude for $C$ is either the TLS density $P$ or phonon coupling $\gamma$ is enhanced, caused by $\mathrm{H}_{2}$ adsorption. As mentioned before in Ref. [29], $T_{\text {co }}$ in bulk Pd polycrystals are very low. The standard TLS models predict a frequency scaling relation $\omega \sim \gamma^{2} T_{\mathrm{co}}^{3}[14,35]$. Since we are comparing devices close to $\sim 20 \mathrm{MHz}$ frequency, the lowering of $T_{\mathrm{co}}$ in our case implies enhancement of $\gamma$ the TLS-phonon coupling constant. As discussed earlier in Ref. [29], with annealed Ta and $\mathrm{Pt}$, the increase of $T_{\mathrm{co}}$ is inferred as $\gamma$ lowering (possibly due to additional tensile stress induced in the system). We can conclude we are effectively detuning the tensile stress to lower values by adding $\mathrm{H}_{2}$, thereby enhancing $\gamma$.

One universal feature of TLS in amorphous systems is that the value of $C$ is in the range $C \sim 10^{-4}-10^{-3}$. This applies to a diversity of systems, such as silica [10], thin films [27], and submicron aluminium beams [25]. Gold nanomechanical resonators showed a slightly lower value, $C \sim 2.5 \times 10^{-5}$ [24]. The clear exception to this is stressed silicon nitride, where $C \sim 10^{-6}$. One classic example of this universality is the similarity between PMMA, a soft glass, and silica, a hard glass. Both PMMA and silica show a similar constant, $C \sim 10^{-4}$, due to commensurate TLS parameters in the expression for $C$ [30]. The fact that we see softening of $\mathrm{Pd}$ as well as reduction of $T_{\mathrm{co}}$ but similar values of $C$ obtained from $\left(\frac{d f}{f_{0}}\right)$ or $Q^{-1}$ above $T_{\text {co }}$ is analogous to the similarity of TLS between PMMA and silica. Surprisingly, the ratio of slopes of frequency shift above and below $T_{\text {co }}$ is $\sim 1.43$ and $\sim 1.5$ for the low $\mathrm{H}_{2}$ samples in Fig. 6(b), reckoning a behavior similar to amorphous dielectrics.

In the low- $\mathrm{H}_{2}$ case [Fig. 6(a)], at temperatures below $200 \mathrm{mK}$, one sees a saturation in dissipation. Such a feature has been predicted [37] as a possible super-radiant phonon emission. In some older experiments [24], where the temperature was corrected using the high-temperature logarithmic frequency shift as a thermometer [38], the feature merged into the power law indicative of thermal decoupling, and these features were at much lower temperatures [24]. Such a correction also did not remove the feature as seen in Fig. 6(a). The sample with higher $\mathrm{H}_{2}$ Fig. 7(a) shows the same feature starting earlier at $\sim 300 \mathrm{mK}$ down to $\sim 250 \mathrm{mK}$, where we could get the data without any need for corrections to the frequency shift. In Ref. [37], two characteristic loss 


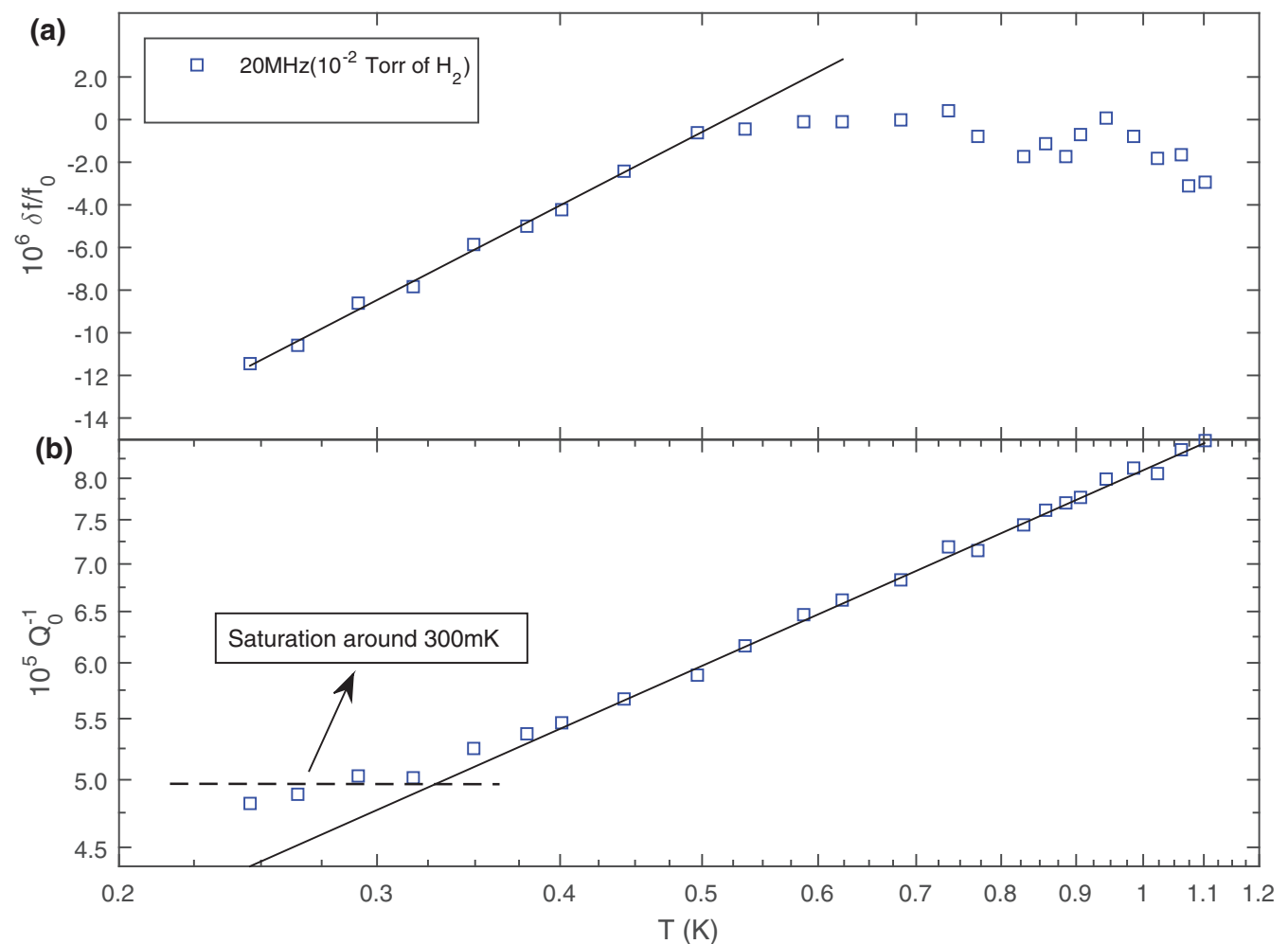

FIG. 7. Sample B1 exposed to $\sim 10^{-2}$ Torr $\mathrm{H}_{2}$ during cool-down. (a) $\left(\frac{d f}{f_{0}}\right)$ as a function of temperature. A $\log$ fit below $T \sim 600 \mathrm{mK}$ yields the constant $C$ with value of $1.54 \times 10^{-5}$. (b) The dissipation data $Q^{-1}$ with a sublinear fit with $\sim T^{0.43}$ is shown as a guide. The low-temperature saturation occurs at $T \sim 300 \mathrm{mK}$. In the data range shown, there is also no thermalization issues. There is no deviation from logarithmic fit to frequency shift at low temperatures.

mechanisms are added $Q_{\text {total }}^{-1}=Q_{0}^{-1}+Q_{\text {pump }}^{-1}$, where $Q_{0}^{-1}$ is the TLS mechanism and $Q_{\text {pump }}^{-1}$ describes a cooperative phonon emission by a small population of TLS sampled within a phonon wavelength. The effect is a weak renormalization of TLS relaxation time $\tau$ by $\tau / N$, where $N$ is the number of TLS that are cooperatively excited by a phonon. The effect is expected only at very low temperatures due to crossover to nonlinear TLS-phonon coupling. The high-temperature plateau is different with $\omega \tau \sim 1$ marking the onset of inelastic processes exciting a spectrum of TLS relaxation rates. When already in the limit $\omega \tau>1$, we expect coherent excitation. One of the criterion for the pumping [37] is the asymmetry energy $\Delta_{0} \leqslant \gamma \epsilon_{o}$, where $\epsilon_{o}$ is some built-in strain. We cannot comment on the static strain status of our devices at cryogenic temperatures, but devices exposed to $\mathrm{H}_{2}$ showed some buckling when warmed up. As mentioned before, the power to drive the system in linear regime was significantly lower with exposure to hydrogen. We also inferred $\gamma$ to be enhanced from the drop in $T_{\text {co }}$ with $\mathrm{H}_{2}$ exposure. The probability to probe more TLS by a phonon is also enhanced in a compressively strained lattice. Other possible scenarios, such as modification of TLS distribution or interactions, are expected at ultra-low temperatures, typically below $10 \mathrm{mK}$ [10]. In our data, the small frequency shift corrections in Fig. 6(a) or none in Fig. 7 for the sample with higher $\mathrm{H}_{2}$ concentration points toward a super-radiant phonon loss as the most plausible scenario.
The tuning of the crossover temperature is reminiscent of the behavior of spin glasses or rather pseudospin glasses in our case. The AC susceptibility of spin glasses shows a cusp indicating a crossover regime when $\omega \tau \sim 1$; i.e., spins are able to follow the ac perturbation $(\omega \tau<1)$ on one side and are unable to follow it on the lower side $(\omega \tau>1)$. As one increases the frequency, one does not see an appreciable shift in the cusp, indicating a slowing down of spins. In conclusion, we have been able to tune TLS scenarios with compressive strain stemming from incorporation of $\mathrm{H}_{2}$. Analogous to ferromagnetic impurities deciding spin-glass temperatures by tuning of magnetic interactions, the role of $\mathrm{H}_{2}$ is to tune a pseudospin interaction by stress. The compressive stress is shown to enhance the phonon-TLS coupling. Softening of the material with $\mathrm{H}_{2}$ has also led to possible super-radiant TLS-phonon losses. The system has the potential to provide further information on understanding dissipation in metallic NEMS.

\section{ACKNOWLEDGMENTS}

A.V. thanks DST (India) Nanomission Project No. SR/NM/NS-1098/2011, DST (India) Ramanujan Fellowship Project No. SR/S2/RJN-26/2010, and IISER (India) at Mohali for funds. We also thank DST (India) for Inspire Fellowships and CSIR-UGC (India) for funding students. We thank Mrs. C. Linz and Dr. J. Eroms for help with HF etching and Mr. Inderjit 
Singh for help in the SEM facility at IISER. A.V. thanks Prof. A. K. Raychaudhuri for several insightful discussions. We acknowledge critical review of the manuscript by Prof. J. R.
Owers-Bradley, Prof. A. D. Armour, Dr. A. Huettel, and Dr. P. Balanarayan. We thank Dr. S. Goyal for some discussions on super-radiance.
[1] K. C. Schwab and M. L. Roukes, Phys. Today 58(7), 36 (2005).

[2] E. M. Weig, R. H. Blick, T. Brandes, J. Kirschbaum, W. Wegscheider, M. Bichler, and J. P. Kotthaus, Phys. Rev. Lett. 92, 046804 (2004).

[3] G. Zolfagharkhani, A. Gaidarzhy, P. Degiovanni, S. Kettemann, P. Fulde, and P. Mohanty, Nature Nanotechnol. 3, 720 (2008); A. G. Malshukov, C. S. Tang, C. S. Chu, and K. A. Chao, Phys. Rev. Lett. 95, 107203 (2005).

[4] K. L. Ekinci and M. Roukes, Rev. Sci. Instrum. 76, 061101 (2005).

[5] X. M. H. Huang, X. L. Feng, C. A. Zorman, M. Mehregany, and M. L. Roukes, New J. Phys. 7, 247 (2005); X. M. H. Huang, C. A. Zorman, M. Mehregany, and M. L. Roukes, Nature 421, 496 (2003).

[6] A. Gaidarzhy, G. Zolfagharkhani, R. L. Badzey, and P. Mohanty, Phys. Rev. Lett. 94, 030402 (2005).

[7] J. D. Teufel, J. W. Harlow, C. A. Regal, and K. W. Lehnert, Phys. Rev. Lett. 101, 197203 (2008); T. Rocheleau, T. Ndukum, C. Macklin, and J. B. Hertzberg, A. A. Clerk, and K. C. Schwab, Nature 463, 72 (2010).

[8] A. D. O'Connell, M. Hofheinz, M. Ansmann, Radoslaw C. Bialczak, M. Lenander, Erik Lucero, M. Neeley, D. Sank, H. Wang, M. Weides, and J. Wenner, J. M. Martinis, and A. N. Cleland, Nature 464, 697 (2010).

[9] L. G. Remus, M. P. Blencowe, and Y. Tanaka, Phys. Rev. B 80, 174103 (2009).

[10] A. D. Fefferman, R. O. Pohl, A. T. Zehnder, and J. M. Parpia, Phys. Rev. Lett. 100, 195501 (2008).

[11] R. C. Zeller and R. O. Pohl, Phys. Rev. B 4, 2029 (1971).

[12] C. H. Enss and R. Hunklinger, Low Temperature Physics (Springer-Verlag, Berlin-Heidelberg, 2005).

[13] P. Esquinazi (ed.), Tunneling Systems in Amorphous and Crystalline Solids (Springer-Verlag, Berlin, 1998).

[14] S. Hunklinger and A. K. Raychaudhuri, Progress in Low Temperature Physics, Vol. IX, edited by H. Brewer (Elsevier, New York, 1986).

[15] P. W. Anderson, B. I. Halperin, and C. M. Varma, Philos. Mag. 25, 1 (1972).

[16] D. R. Southworth, R. A. Barton, S. S. Verbridge, B. Ilic, A. D. Fefferman, H. G. Craighead, and J. M. Parpia, Phys. Rev. Lett. 102, 225503 (2009).

[17] A. J. Leggett and D. C. Vural, J. Phys. Chem. B 117, 12966 (2013).
[18] C. C. Yu, J. Low Temp. Phys. 137, 251 (2004).

[19] X. Liu, B. E. White, Jr., R. O. Pohl, E. Iwanizcko, K. M. Jones, A. H. Mahan, B. N. Nelson, R. S. Crandall, and S. Veprek, Phys. Rev. Lett. 78, 4418 (1997).

[20] X. Liu, D. R. Queen, T. H. Metcalf, J. E. Karel, and F. Hellman, Phys. Rev. Lett. 113, 025503 (2014).

[21] M. Imboden and P. Mohanty, Phys. Rep. 534, 89 (2014).

[22] K. J. Lulla, M. Defoort, C. Blanc, O. Bourgeois, and E. Collin, Phys. Rev. Lett. 110, 177206 (2013).

[23] T. F. Li, Y. A. Pashkin, O. Astafiev, Y. Nakamura, J. S. Tsai, and H. Im, Appl. Phys. Lett. 92, 043112 (2008).

[24] A. Venkatesan, K. J. Lulla, M. J. Patton, A. D. Armour, C. J. Mellor, and J. R. Owers-Bradley, Phys. Rev. B 81, 073410 (2010); A. Venkatesan, K. J. Lulla, M. J. Patton, A. D. Armour, C. J. Mellor, and J. R. Owers-Bradley, J. Low Temp. Phys. 158, 685 (2010).

[25] F. Hoehne, Y. A. Pashkin, O. Astafiev, L. Faoro, L. B. Ioffe, Y. Nakamura, and J. S. Tsai, Phys. Rev. B 81, 184112 (2010).

[26] J. Sulkko, M. A. Sillanp, P. Hkkinen, L. Lechner, M. Helle, A. Fefferman, J. Parpia, and P. J. Hakonen, Nano Lett. 10, 4884 (2010).

[27] X. Liu, E. J. Thompson, B. E. White, and R. O. Pohl, Phys. Rev. B 59, 11767 (1999).

[28] A. D. Fefferman, R. O. Pohl, and J. M. Parpia, Phys. Rev. B 82, 064302 (2010).

[29] E. Gaganidze and P. Esquinazi, J. Phys. IV (France) 06, C8-515 (1996).

[30] J. Wu and C. C. Yu, Phys. Rev. B 84, 174109 (2011).

[31] X. M. H. Huang, M. Manolidis, S. C. Jun, and J. Hone, Appl. Phys. Lett. 86, 143104 (2005).

[32] A. Cleland and M. L. Roukes, Sens. Actuat. 72, 256 (1999).

[33] J. Atalaya, A. Isacsson, and M. I. Dykman, Phys. Rev. Lett. 106, 227202 (2011).

[34] A. Dayo, W. Alnasrallah, and J. Krim, Phys. Rev. Lett. 80, 1690 (1998).

[35] A. K. Raychaudhuri and S. Hunklinger, Z. Phys. B 57, 113 (1984).

[36] C. Seoanez, F. Guinea, and A. H. Castro Neto, Phys. Rev. B 77, 125107 (2008).

[37] K. H. Ahn and P. Mohanty, Phys. Rev. Lett. 90, 085504 (2003).

[38] J. Classen, C. Enss, and S. Hunklinger, Phys. Rev. Lett. 86, 2480 (2001). 